\title{
Incidencia de la autorregulación del aprendizaje en las competencias lectoras*
}

\author{
ERICA YASMÍN PINZÓN SANTOS** \\ MYRIAM SORAYA SUÁREZ ROJAS*** \\ FRANCISCO CONEJO CARRASCO ${ }^{* * * *}$
}

Recepción: 03 de diciembre de 2020

Aprobación: 28 de marzo de 2021

Forma de citar este artículo: Pinzón, E. Suárez, M \& Conejo, F. M (2021). Incidencia de la autorregulación del aprendizaje en las competencias lectoras. Cuadernos de Lingüística Hispánica, (37), e12152

\section{(60) https://doi.org/10.19053/0121053X.n37.2021.12152}

* Artículo de investigación, producto derivado del proyecto de investigación denominado: "Influencia de los componentes de la autorregulación del aprendizaje en las competencias lectoras" - Maestría en Educación. Corporación Universitaria Minuto de Dios- UNIMINUTO.

** Magíster en Educación. Corporación Universitaria Minuto de Dios-UNIMINUTO. Licenciada en Lengua Castellana y Comunicación. Universidad de Pamplona. Colombia. ericajazminpin@hotmail.com. https:/orcid. org/0000-0002-8895-0451

*** Magíster en Educación. Psicóloga. Lic. en Educación. Docente investigadora. Asesora proyecto de investigación sub- línea de investigación Autorregulación del Aprendizaje. Corporación Universitaria Minuto de DiosUNIMINUTO.myriam.suarez-r@uniminuto.edu.co sorayasuarez73@gmail.com. @https://orcid.org/0000$\underline{0001-7856-4226}$

****Doctorando en Investigación Transdisciplinar en Educación. Máster en Educación. Licenciado en Historia. Investigador Junior de MINCIENCIAS. Líder de la sub-línea de investigación Autorregulación del Aprendizaje. Corporación Universitaria Minuto de Dios-UNIMINUTO. francisco.conejo@uniminuto.edu , fconejocarr@ uniminuto.edu.co. https://orcid.org/0000-0002-3490-1937 


\section{Resumen}

Este artículo surge en un contexto social altamente vulnerable, con problemas sociales y económicos, en medio de una emergencia sanitaria por el coronavirus SARS-CoV-2. Como metodología se adopta el enfoque mixto para aprovechar el valor del enfoque cualitativo y cuantitativo de una manera descriptiva. Se utilizan técnicas de recolección de datos como cuestionario con escala de Likert, entrevistas en grupos focales y observación participante, aplicadas a estudiantes de secundaria. Los hallazgos permiten mejorar la mediación entre el saber y los estudiantes a partir de la autorregulación en las prácticas lectoras con elementos como la volición, la autorreflexión y la motivación, puesto que aprender de manera significativa implica que el estudiante sea consciente de su rol; además, el manejo de sus pensamientos, emociones y acciones le permite el desarrollo de sus habilidades lectoras.

Palabras clave: autorregulación del aprendizaje, competencias lectoras, perspectiva volitiva de la autorregulación, autorreflexión del aprendizaje, motivación intrínseca y extrínseca, estrategias de comprensión lectora.

\section{Impact of Self-Regulated Learning in Reading Skills}

\section{Abstract}

This article arises in a highly vulnerable social context, with social and economic problems, in the midst of a health emergency due to the SARS-CoV-2 coronavirus. As a methodology, the mixed approach is adopted to take advantage of the value of qualitative and quantitative approach in a descriptive way. Data collection techniques are used such as a Likert scale questionnaire, focus groups interviews and participant observation, applied to high school students. Findings allow improving the mediation between knowledge and students based on self-regulation in reading practices with elements such as volition, self-reflection, and motivations, since learning in a meaningful way implies that the students are aware of their role; In addition, the handling of their thoughts, emotions and actions allows them to develop their reading skills.

Keywords: self-regulated learning, reading skills, volitional perspective of self-regulation, self-reflective learning, intrinsic and extrinsic motivation, reading comprehension strategies. 


\section{L'impact de l'autorégulation de l'apprentissage sur le compétences en lecture}

\section{Résumé}

Cet article s'inscrit dans un contexte social très vulnérable, avec des problèmes sociaux et économiques, au milieu d'une urgence sanitaire due au coronavirus SRASCoV-2. En tant que démarche méthodologique, l'approche mixte est adoptée pour tirer parti de la valeur de l'approche qualitative et quantitative de manière descriptive. Des techniques de quête de données sont utilisées, telles que le questionnaire à l'échelle de Likert, les entretiens de groupe et l'observation participante, appliquées aux élèves du secondaire. Les résultats permettent d'améliorer la médiation entre les connaissances et les élèves à partir de l'autorégulation dans les pratiques de lecture avec des éléments tels que la volition, l'autoréflexion et la motivation, puisqu'apprendre de manière significative implique que l'élève soit conscient de son rôle; de plus, la gestion de ses pensées, émotions et actions permet de développer ses compétences en lecture.

Mots clés: autorégulation de l'apprentissage, compétences en lecture, perspective volitive de l'autorégulation, auto-réflexion de l'apprentissage, motivation intrinsèque et extrinsèque, stratégies de compréhension de la lecture

\section{Impacto da auto-regulação da aprendizagem nas competências de leitura}

\section{Resumo}

Este artigo surge em um contexto social de alta vulnerabilidade, com problemas sociais e econômicos, em meio a uma emergência sanitária devido ao coronavírus SARS-CoV-2. Como metodologia, a abordagem mista é adotada para aproveitar o valor da abordagem qualitativa e quantitativa de forma descritiva. São utilizadas técnicas de coleta de dados, como questionário em escala Likert, entrevistas com grupos focais e observação participante, aplicados a alunos do ensino médio. Os resultados permitem melhorar a mediação entre o conhecimento e os alunos com base na auto regulação em práticas de leitura com elementos como vontade, autorreflexão e motivação, pois 0 aprendizado de uma forma significativa implica que o aluno está ciente do seu papel; além disso, o gerenciamento de seus pensamentos, emoções e ações permite que desenvolvam suas habilidades de leitura.

Palavras-chave: auto regulação da aprendizagem, habilidades de leitura, perspectiva volitiva de auto regulação, autorreflexão da aprendizagem, motivação intrínseca e extrínseca, estratégias de compreensão de leitura. 


\section{Introducción}

Para responder a las necesidades encontradas en el área de lenguaje, se buscan mecanismos que mejoren la comprensión lectora mediante la autorregulación, vista como el proceso que permite construir los propios conocimientos, dando sentido a lo que se aprende, donde el protagonista es el estudiante.

En la profesión docente surgen a diario interrogantes acerca de cómo mejorar el proceso enseñanza-aprendizaje. Hoy en día, gracias a la globalización y a los medios de comunicación, la educación trasciende fronteras, de manera que se pueden conocer las prácticas, las estrategias y los recursos que utilizan diferentes profesionales para apoyar la gestión; sin embargo, cada contexto es único y a veces es necesario crear estrategias propias.

Por esta razón, esta investigación se desarrolla a partir del siguiente interrogante: ¿Cómo influyen los componentes de la autorregulación del aprendizaje en las competencias lectoras de los estudiantes de grado octavo? Se utiliza un enfoque mixto en el que se valoran pensamientos, sentimientos y acciones propias del estudiante frente al proceso lector, siguiendo la línea denominada autorregulación del aprendizaje, y se plantea como objetivo describir la influencia de los componentes de la autorregulación del aprendizaje en las competencias lectoras de los estudiantes de octavo grado.

Para este estudio se toman los postulados de Zimmerman y Moylan, descritos por Panadero y Tapias (2014) al definir la autorregulación como "el control que el sujeto realiza sobre sus pensamientos, acciones, emociones y motivación a través de estrategias personales para alcanzar los objetivos que ha establecido" (pp. 450- 451).

Con base en esto y aplicado a la competencia lectora, vista como la capacidad de comprender y aplicar los conocimientos adquiridos en el ámbito social, Solé (1992) expone:

Comprender es ante todo un proceso de construcción de significados acerca del texto que pretendemos comprender. Es un proceso que implica activamente al lector, en la medida que la comprensión que realiza no es un derivado de la recitación del contenido de que se trata. Para ello es indispensable que el lector encuentre sentido en efectuar el esfuerzo cognitivo que supone leer, lo que exige conocer qué va a leer, y para qué va a hacerlo; exige además disponer de recursos - conocimiento previo relevante, confianza en las propias posibilidades como lector, disponibilidad de ayudas necesarias. (p. 37)

En este sentido, y como fundamento de esta investigación, tomamos prestado lo enunciado por Panadero y Tapias (2014): "El problema al que nos enfrentamos es averiguar cómo podemos ayudar a los alumnos a afrontar su aprendizaje de forma intencional, autónoma y efectiva, proceso denominado autorregulación” (p. 450). Y para esto se muestra la importancia del docente como investigador y mediador, 
se promueven estrategias que motiven al estudiante a querer aprender, a hacer uso de sus conocimientos previos, de su experiencia, de su anhelo de progreso, bajo principios de libertad y autonomía.

Además del avance de los aprendizajes de los estudiantes a través de la autorregulación, se pretende contribuir con el progreso del sistema educativo y la eficiencia del sector, transformando la educación desde su eje principal, "el educando", pues se cree que cuando este logra avanzar en su conocimiento, reconoce su papel dentro de la sociedad, asume responsabilidades colectivas y construye sociedades más justas basadas en la identidad y libertad.

El siglo XXI está lleno de transformaciones, por ende, la educación no se debe quedar estancada, sino todo lo contrario, estar a la vanguardia de los cambios globales. Se requiere que conocimientos, habilidades, actitudes y valores que se desarrollan en las personas mediante la interacción con los demás, permitan analizar, comprender, interactuar, proponer estrategias para el mundo actual. Radica aquí la importancia de la investigación para la innovación y la creatividad en la enseñanza-aprendizaje.

La investigación parte de la autorregulación, vista como la toma de conciencia acerca de la necesidad y de querer aprender, de conocer, de ser cada vez más diestro al pensar o al realizar una acción. No todos los seres humanos son conscientes de sus necesidades intelectuales, pues algunos reducen su pensamiento a las necesidades físicas. La autorregulación permite dirigir las acciones, el esfuerzo y la dedicación hacia el logro de los objetivos propuestos.

La autorregulación implica tener conciencia del propio pensamiento, es el conocimiento acerca de cómo se aprende. Este modo más profundo de aprendizaje se desarrolla a través de observar en acción las propias conductas adoptadas para aprender. Además de observar, el sujeto vigila y controla sus comportamientos para obtener un aprendizaje más efectivo. (Crispín et al., 2011, p. 49)

Adquirir conocimientos le permite al estudiante no solo prepararse para una vida laboral sino formar su identidad, saber diferenciar lo que está bien de lo que está mal, mejorar las diferentes situaciones de vida y crear oportunidades de progreso. Se dice que el saber le otorga al ser humano libertad para actuar, pensar y ser.

Dentro de la autorregulación se encuentra el autocontrol, elemento que le permite al estudiante reconocer, valorar y potenciar sus emociones, sentimientos y acciones frente a una meta. Razón por la que se consideran de gran importancia las enseñanzas de Zimmerman y Moylan (2009, citados por Panadero \& Tapia, 2014) sobre el modelo cíclico, el cual está compuesto de tres fases: planeación, ejecución, autorreflexión; donde termina una, inicia la otra. Y se ven como el antes, el durante y el después de haber adquirido ciertos saberes. Lo que muestra de manera implícita cómo el ser humano nunca termina de aprender, el ciclo no tiene 
un final. De igual forma sucede con el conocimiento, que es infinito y proviene de la exploración, la interacción y la fundamentación.

Con base en esto y articulándolo con la parte lingüística desde la competencia lectora donde el estudiante lee para aprender, divertirse, cultivar su autonomía, imaginación y creatividad, se toma lo descrito por la OECD (2019), "La competencia lectora consiste en comprender, usar, evaluar, reflexionar e interactuar con los textos; con el fin de lograr los objetivos propios, desarrollar el conocimiento y el potencial propios y participar en sociedad" (p. 28). Leer implica comprender, nunca se puede considerar este proceso como algo mecánico, dado que se requiere que el lector entienda el significado de las palabras para que interprete el mensaje y así sea un proceso significativo.

Según Jiménez (2014), las actividades curriculares establecen relaciones entre lo que se lee, los conocimientos previos y el deseo de construir nuevos significados. Por ello, en las instituciones educativas permanentemente se enuncia la comprensión lectora como la fórmula para que niños y jóvenes entiendan lo que leen y de esta forma mejoren su rendimiento académico y su valoración en los procesos evaluativos; pero la comprensión lectora implica no solo destrezas lingüísticas sino también metacognitivas, en las que el lector debe estar inmerso en el proceso de manera autónoma, pues solo de esta forma podrá llegar a la decodificación y construcción de significados.

Bustamante (2015) manifiesta que, a pesar de que el Gobierno está haciendo esfuerzos para mejorar la calidad educativa en Colombia, los resultados en las Pruebas Saber no muestran progreso, sino todo lo contrario, situación que aumenta la brecha que hay con otros países.

Por ser la lectura de gran importancia dentro del proceso de enseñanzaaprendizaje, la comprensión lectora implica ser trabajada en todas las áreas del conocimiento.

Al respecto, Vargas (2001) menciona:

La baja motivación hacia la lectura es lo cotidiano en nuestra labor pedagógica. Muchas son las variables que influyen en ese fenómeno y muchas las investigaciones que se han hecho al respecto. Tenemos el diagnóstico: a muchos alumnos no les agrada leer. La verdad es que no son pocos los adultos, entre ellos algunos docentes, que tienen la misma actitud. Ante este problema, ¿tenemos soluciones desde la escuela? ¿Realmente le permitimos al niño 0 al joven que se implique activamente en la búsqueda del significado? En el proceso de la lectura hay dos momentos bien importantes: el primero, cuando se aprende a leer; el segundo, cuando se aprende leyendo. (p. 24) 


\section{Metodología}

Tomando como referencia a Hernández et al. (2014) en su obra Metodología de la investigación, donde sostienen que los proyectos de investigación pueden estar fundamentados desde diferentes enfoques, se adoptó en esta investigación el enfoque mixto a fin de aprovechar el valor del enfoque cualitativo y cuantitativo de una manera descriptiva.

Hernández et al. (2014) brindan amplia información sobre el enfoque cualitativo cimentado en la interpretación, el entendimiento del porqué de las acciones, a través de instrumentos como grupos focales, biografías, observación, entre otros. También hablan sobre el enfoque cuantitativo y demuestran el valor de los datos estadísticos recolectados a través de cuestionarios cerrados, pruebas estandarizadas, entre otros.

Así que, con base en lo anterior, en este estudio se utilizan instrumentos de interacción y participación para evaluar el desarrollo natural de los sucesos mediante la interpretación centrada en las acciones, argumentaciones y descripciones brindadas por los estudiantes, el investigador y por el proceso de enseñanza- aprendizaje.

Por consiguiente, las técnicas y los instrumentos mediante los cuales se recolecta la información fueron aplicados en el siguiente orden:

1. Cuestionario para conocer las opiniones y actitudes de los estudiantes en cuanto a su volición y motivación. Este se presenta según la escala de Likert, que da fiabilidad y validez a los resultados expuestos en un análisis estadístico.

2. Entrevista en grupos focales (10 estudiantes). Según Hamui y Varela (2012), este instrumento permite ver la realidad de la interacción social y la mediación con el aprendizaje a través de los pensamientos, los sentimientos y las vivencias de los estudiantes, expresados de manera fluida y espontánea.

3. Observación participante. El investigador interactúa con los estudiantes en el escenario y ambiente de trabajo. Para esto se utiliza la participación, la observación y la interrogación, dejando registro en un diario de campo. Con esto se pretende mostrar los resultados en el uso de estrategias de lectura mediadas por la autorregulación.

El análisis de datos se realiza de manera secuencial, describiendo a través del enfoque mixto los resultados obtenidos, donde se evalúa e interpreta la información dentro del contexto real, la cual es de carácter objetivo y subjetivo. Según Hernández et al. (2014), "La recolección de datos ocurre en los ambientes naturales y cotidianos de los participantes o unidades de análisis. En el caso de seres humanos, en su vida diaria: cómo hablan, en qué creen, qué sienten, cómo piensan, cómo interactúan” 
(p. 409). El enfoque mixto desde lo cuantitativo permite de manera sistemática el estudio de la realidad apoyado en el análisis estadístico, mediante planteamientos porcentuales, donde se mide de manera objetiva el resultado, para determinar el margen del interrogante planteado, lo que hace más confiable la investigación.

\section{Método de investigación}

Al pretender hallar los elementos reguladores predominantes en la comprensión lectora de un grupo de jóvenes en edades entre 13 y 17 años, se opta por el diseño metodológico descriptivo, donde los estudiantes son observados dentro de su contexto educativo natural, a fin de identificar las relaciones existentes entre las variables.

Ahora bien, Arias (2012) define la investigación descriptiva como "caracterización de un hecho, fenómeno, individuo o grupo, con el fin de establecer su estructura o comportamiento. Los resultados de este tipo de investigación se ubican en un nivel intermedio en cuanto a la profundidad de los conocimientos" (p. 24). Vale decir que este proceso se va a seguir dentro de la investigación.

En este estudio se utilizaron datos secundarios como lo son los enunciados en el marco referencial, dado que muestran una visión del conocimiento obtenido hasta el momento. Partiendo de allí y adaptándolo al contexto sociocultural y demográfico de la población, se inicia esta investigación.

\section{Población y muestra de la investigación}

La población objeto de esta investigación corresponde a 97 estudiantes del grado octavo, pertenecientes en un $85 \%$ al nivel 1 del Sisbén, 40 \% es población víctima del conflicto armado. La principal actividad económica del sector es el comercio informal, la construcción, el trabajo de campo y las labores domésticas. La muestra objeto de la investigación es un grupo de 26 estudiantes con edades entre 13 y 17 años.

\section{Resultados}

Como el estudiante es el eje principal en el proceso de aprendizaje, es necesario impulsar su autorregulación frente al manejo de sus pensamientos, emociones y acciones, de modo que le permita el desarrollo pleno de sus habilidades lectoras.

La investigación señala que elementos como la voluntad y la autorreflexión están ligados a la motivación, y estos, al proceso lector; de allí la importancia de enseñar de forma motivadora, para que estos aprendizajes tengan significado en el proceso de formación de los jóvenes.

Esta influencia se puede observar a partir de cuatro categorías: volición, autorreflexión, motivación y estrategias lectoras. 
En la identificación de la volición que tienen los estudiantes de octavo grado en su proceso de comprensión lectora, se infiere que la volición depende de sus gustos, de otra forma pocos realizan la acción de manera voluntaria.

En cuanto a determinar la incidencia de la autorreflexión en el desarrollo de competencias lectoras de los estudiantes de octavo grado durante el primer semestre del 2020, se deduce, de acuerdo con los resultados, que este componente permite al estudiante autoevaluarse, y se aclara que el proceso lector depende del gusto por el libro que se va a leer, la curiosidad y la intención. Durante esta fase, el estudiante observa y analiza su rendimiento, su participación como eje principal en el proceso de aprendizaje, y expone sus argumentos frente a los resultados obtenidos.

Al establecer las estrategias de control motivacional que mejoran la actividad lectora en los estudiantes de octavo grado, se observa que la motivación se involucra en todo el proceso. En el grupo participante se acentúa la falta de motivación extrínseca. Como estrategia frente a sus necesidades se requieren libros ajustados a sus intereses, para evitar que este factor obstaculice su práctica lectora. La motivación intrínseca se ve fortalecida cuando los estudiantes superan los errores, convirtiéndolos en un reto que los motiva de manera personal. Así mismo, terminar la lectura y entenderla produce sensación de éxito, según lo evidenciaron los participantes.

Frente al planteamiento de estrategias de lectura que, mediadas por la autorregulación, permitan desarrollar competencias de comprensión lectora en los estudiantes de octavo grado, en la investigación se utilizaron las señaladas por Kenneth Goodman (1982): muestreo, predicción, inferencia, confirmación, corrección, que permitieron incentivar la curiosidad y llevar al estudiante a alcanzar los objetivos propuestos, de manera dinámica y significativa.

\section{Respuesta a la pregunta de investigación y objetivos}

¿Cómo influyen los componentes de la autorregulación del aprendizaje en las competencias lectoras de los estudiantes de octavo grado de la Institución Técnica Empresarial Llano Lindo, durante el primer semestre del 2020?

Aprender de manera significativa implica que el estudiante sea consciente de su rol. El manejo de sus pensamientos, emociones y acciones le permite el desarrollo de sus habilidades lectoras. Mediante esta investigación se confirma que componentes como la voluntad y la autorreflexión están ligados a la motivación, de allí la importancia de que todos los docentes reconozcan cómo orientar de manera motivadora, para que estos aprendizajes tengan significado en el proceso de formación de los jóvenes. Torrano y González (2004) lo confirman al enunciar: "la educación debe ayudar a los alumnos a ser conscientes de su pensamiento, a ser estratégicos y a dirigir su motivación hacia metas valiosas" (p. 2), proceso en el que el estudiante aprende de manera autónoma y en el que la práctica autorreflexiva es esencial, donde se tiene claro que aprender es una oportunidad y no una obligación. 
Esto requiere orientar al joven hacia aprender a aprender, proceso en el que factores como la voluntad, la autorreflexión y la motivación influyen de manera activa y conjunta dentro de la autorregulación del aprendizaje en la comprensión lectora de los estudiantes de octavo grado, según se observa a continuación.

Frente al objetivo general de describir la influencia de los componentes de la autorregulación del aprendizaje en las competencias lectoras de los estudiantes de octavo grado, se halló que los problemas suscitados en el proceso lector se pueden resolver cuando el estudiante toma conciencia de su rol, tiene voluntad, siente agrado por el proceso y por último autorreflexiona acerca de su aprendizaje; para esto es indispensable la motivación, no solo al inicio, sino durante todo el proceso. Según Santrock (2002), la motivación es "el conjunto de razones por las que las personas se comportan de las formas en que lo hacen. El comportamiento motivado es vigoroso, dirigido y sostenido" (p. 432).

De manera que, elementos de la autorregulación descritos por Zimmerman y Moylan (2009, citados por Panadero \& Tapia, 2014) como la voluntad, la autorreflexión y la motivación, le permiten al estudiante apropiarse de su proceso lector para alcanzar los objetivos propuestos, antes, durante y después de la lectura. Por tanto, el dominio de estos componentes de la autorregulación del aprendizaje promueve la conciencia y la proyección de emociones y acciones en la construcción de una comprensión e interpretación lectora.

Con esta investigación se observó que la motivación que tienen los estudiantes de octavo grado frente al proceso lector afecta de manera positiva o negativa el proceso, que variables como la voluntad y la autorreflexión son influenciadas por la motivación, que es un canal para conseguir el aprendizaje significativo. Se puede también interpretar la necesidad de potenciar la curiosidad, como factor motivacional de alta relevancia, dado que impulsa a los jóvenes a querer leer.

Siempre se ha escuchado que la lectura despierta la curiosidad; con el trabajo realizado se interpreta que la curiosidad lleva a la lectura y por ende al logro de los objetivos. Al ser el conocimiento infinito, la curiosidad de los jóvenes puede alimentarse continuamente para llevarlos a querer autorregular su aprendizaje.

\section{Resultados obtenidos en la aplicación de los instrumentos}

En el cuestionario planteado según escala Likert se trazaron dos rutas importantes para identificar la volición que tienen los estudiantes de octavo grado frente a su proceso de comprensión lectora: primero, la autobservación, donde los estudiantes analizan sus pensamientos y acciones, y se halló que pocos estudiantes leen por decisión propia pues carecen de ese esfuerzo intencional a pesar de que sienten gusto por la lectura y dedican tiempo libre a esta. 
Tabla 1. Volición: autobservación en estudiantes de $8 .^{\circ}$

\begin{tabular}{lcccc}
\hline & Siempre & Ocasionalmente & Casi nunca & Nunca \\
$\begin{array}{l}\text { Dedico tiempo libre a la lectura } \\
\begin{array}{l}\text { Administro bien mí tiempo, preparando } \\
\text { mis tareas escolares oportunamente. }\end{array}\end{array}$ & $35 \%$ & $42 \%$ & $19 \%$ & $4 \%$ \\
$\begin{array}{l}\text { Cuando leo lo hago porque me agrada } \\
\begin{array}{l}\text { Reconozco el valor de la lectura no solo en } \\
\text { mi proceso escolar sino en mi vida. }\end{array}\end{array}$ & $62 \%$ & $46 \%$ & $15 \%$ & $0 \%$ \\
$\begin{array}{l}\text { Reflexiono acerca de mi proceso lector, } \\
\text { como hábito que puede mejorar las } \\
\text { condiciones sociales y humanas. }\end{array}$ & $35 \%$ & $27 \%$ & $11 \%$ & $0 \%$ \\
$\begin{array}{l}\text { Leo solo cuando en el colegio los profesores } \\
\text { me exigen hacerlo. }\end{array}$ & $27 \%$ & $35 \%$ & $15 \%$ & $0 \%$ \\
$\begin{array}{l}\text { Considero que lo importante del plan lector } \\
\text { es obtener una buena calificación. }\end{array}$ & $31 \%$ & $27 \%$ & $23 \%$ & $15 \%$ \\
$\begin{array}{l}\text { Considero el éxito en la lectura es cuestión } \\
\text { de suerte. }\end{array}$ & $16 \%$ & $23 \%$ & $15 \%$ & $46 \%$ \\
\hline
\end{tabular}

Fuente: elaboración propia. Resultados del cuestionario sobre autobservación realizado a 26 participantes en la investigación "Incidencia de la autorregulación del aprendizaje en las competencias lectoras".

Segundo, el autocontrol, definido por Panadero y Tapia (2014) como el proceso para mantener la concentración y el interés a través de estrategias de tipo metacognitivo o motivacional, factor importante en la edad de los participantes, porque tienden a distraerse fácilmente, se frustran frente a actividades consideradas complejas y optan por hacerlas a un lado y verlas con negatividad.

Se concluye que los jóvenes participantes carecen de estrategias al leer, lo que arroja como resultado la falta de autocontrol, la que se considera una causante del bajo nivel lector. Según Panadero y Tapia (2014), "Mantener la concentración y el interés durante la actividad no se consigue sin esfuerzo, al contrario, su mantenimiento requiere del uso de una serie de estrategias y acciones" (p. 455). 
Tabla 2. Volición: autocontrol en estudiantes de $8 .^{\circ}$

\begin{tabular}{lllll}
\hline & Siempre & Ocasionalmente & Casi nunca & Nunca \\
$\begin{array}{l}\text { Tengo estrategias de lectura, } \\
\text { como lo es el subrayado, la } \\
\text { inferencia, la corrección, } \\
\text { entre otras. }\end{array}$ & $8 \%$ & $15 \%$ & $50 \%$ & $27 \%$ \\
$\begin{array}{l}\text { Aunque surjan obstáculos en } \\
\text { cuanto a la adquisición de }\end{array}$ & $50 \%$ & $38 \%$ & $12 \%$ & $0 \%$ \\
$\begin{array}{l}\text { libros, pocos o nulos recursos } \\
\text { interactivos, entre otros, me } \\
\text { las ingenio para alcanzar los } \\
\text { objetivos de aprendizaje. }\end{array}$ & & & & \\
\end{tabular}

Fuente: elaboración propia. Resultados del cuestionario sobre autocontrol realizado a 26 participantes en la investigación incidencia de la autorregulación del aprendizaje en las competencias lectoras.

Por su parte, la entrevista en grupos focales y la observación participante permitieron determinar la incidencia de la autorreflexión en el desarrollo de competencias lectoras de los estudiantes de octavo grado, teniendo en cuenta dos de los componentes más relevantes: el autojuicio y la autorreacción.

El autojuicio, que trata del "proceso mediante el cual el alumno juzga su ejecución de la actividad. Se compone de la autoevaluación y de las atribuciones causales" Panadero y Tapia (2014. p. 457), y la autorreacción, "En la medida en que los alumnos aprendan a valorar sus éxitos y fracasos como ocasiones para mejorar sabiendo cómo reaccionar, pueden controlar las atribuciones que realizan para que están sean adaptativas, controlando así mejor sus emociones" (Panadero \& Tapia, 2014, p. 458).

Con relación al autojuicio, se concluye que la mayoría de los participantes se autoevalúan y coinciden en afirmar que el proceso lector depende de factores como el gusto, la curiosidad y la intención. Los participantes concuerdan al mencionar que lo más difícil de una lectura es dar respuesta acertada a la evaluación realizada por el docente, por tratarse esta de manera tradicional (memorística). Y en la autorreacción, en el desarrollo del libro los jóvenes aluden a que depende de muchos factores como son el lugar, el propósito de la lectura, la motivación, el gusto por el texto o libro.

Lo descrito arroja como resultado que los diferentes estímulos internos y externos son los principales agentes que llevan al joven a la autorreflexión positiva, negativa o neutral en su proceso lector.

En el cuestionario aplicado con escala Likert también se lograron establecer las estrategias de control motivacional que mejoran la actividad lectora en los estudiantes de este grado; se analizó desde la motivación intrínseca valorando factores internos, y desde la motivación extrínseca a partir de los ambientes en su contexto. 
En la motivación intrínseca se concluye que los estudiantes participantes siempre están interesados en la adquisición de nuevas habilidades y en la mejora de sus conocimientos, aun a riesgo de cometer errores, el superarlos se convierte en un reto que los motiva de manera personal. Los participantes ven en la lectura un medio de diversión, de imaginación, sintiendo gusto y agrado; consideran que es beneficioso para la salud mental. Ya en la motivación extrínseca es diferente, pocas veces los jóvenes comparten sus gustos y experiencias lectoras, muy pocos estudiantes reciben de sus padres y amigos libros como un detalle valioso, faltan libros en casa, factor determinante en la lectura de los jóvenes; así que carecer de este elemento implica que las estrategias vayan planteadas a mejorarlo, y para esto hay que tener en cuenta lo que afirman Anaya y Anaya (2010) sobre la motivación extrínseca, la cual es definida por estos autores "como aquella que procede de fuera y que conduce a la ejecución de la tarea", y según la investigación esta requiere mejoras.

Tabla 3. Motivación en estudiantes de $8 .^{\circ}$

\begin{tabular}{|c|c|c|c|c|}
\hline & Siempre & Ocasionalmente & Casi nunca & Nunca \\
\hline $\begin{array}{l}\text { Estoy interesado en la adquisición de nuevas } \\
\text { habilidades y en la mejora de mis } \\
\text { conocimientos, aun a riesgo de cometer } \\
\text { errores. }\end{array}$ & $77 \%$ & $15 \%$ & $4 \%$ & $4 \%$ \\
\hline Considero la lectura me lleva a mundos & $58 \%$ & $27 \%$ & $7 \%$ & $8 \%$ \\
\hline $\begin{array}{l}\text { Al terminar de leer un libro en lugar de } \\
\text { alegrarte, te lamentas porque se acaba la } \\
\text { diversión y querías saber más del desarrollo } \\
\text { de los hechos. }\end{array}$ & $46 \%$ & $31 \%$ & $8 \%$ & $15 \%$ \\
\hline $\begin{array}{l}\text { Me aburre el tener que leer porque considero } \\
\text { muy valioso mi tiempo para dedicarlo a esa } \\
\text { actividad. }\end{array}$ & $0 \%$ & $27 \%$ & $27 \%$ & $46 \%$ \\
\hline $\begin{array}{l}\text { Leer es un beneficioso ejercicio mental que } \\
\text { procuro realizar a diario. }\end{array}$ & $35 \%$ & $46 \%$ & $15 \%$ & $4 \%$ \\
\hline $\begin{array}{l}\text { Comparto mis conocimientos con personas } \\
\text { que tienen mis mismos gustos lectores. }\end{array}$ & $16 \%$ & $19 \%$ & $42 \%$ & $23 \%$ \\
\hline $\begin{array}{l}\text { Los espacios y ambientes con que cuento para } \\
\text { la lectura son óptimos. }\end{array}$ & $42 \%$ & $50 \%$ & $8 \%$ & $0 \%$ \\
\hline $\begin{array}{l}\text { Mis padres y amigos me regalan libros como } \\
\text { algo valioso e importante. }\end{array}$ & $11 \%$ & $23 \%$ & $31 \%$ & $35 \%$ \\
\hline $\begin{array}{l}\text { Prefiero actividades en las que el esfuerzo sea } \\
\text { poco para tener tiempo de ocio. }\end{array}$ & $27 \%$ & $35 \%$ & $23 \%$ & $15 \%$ \\
\hline $\begin{array}{l}\text { Tengo libros en mi casa y los clasifico para } \\
\text { poder acceder a ellos en el momento que lo } \\
\text { prefiera. }\end{array}$ & $16 \%$ & $15 \%$ & $42 \%$ & $27 \%$ \\
\hline
\end{tabular}

Fuente: elaboración propia. Resultados del cuestionario sobre motivación realizado a 26 participantes en la investigación "Incidencia de la autorregulación del aprendizaje en las competencias lectoras". 


\section{Conclusión}

Al plantear estrategias de lectura mediadas por la autorregulación que permitan desarrollar competencias de comprensión lectora en los estudiantes de octavo grado, durante el primer semestre del 2020, se aplicaron las estrategias enunciadas por Kenneth Goodman (1982): muestreo, predicción, inferencia, confirmación, corrección. A partir de allí se concluye que dan buen resultado, siempre y cuando la motivación esté en cada fase del proceso; es así como en el muestreo está implícita la motivación personal reflejada en los gustos e intenciones del estudiante, y de la misma forma la motivación extrínseca cuando se alude a los libros que el estudiante tiene al alcance y los estímulos que recibe de padres, amigos, profesores, entre otros.

Con la predicción y la inferencia se acrecienta la curiosidad, la imaginación y el conocimiento; la confirmación y la corrección se dieron de forma simultánea y permitieron alcanzar los objetivos, es decir, la primera involucraba a la última. En algunos casos, los participantes se adelantaban al proceso, se cree que se debe a la nueva forma de estudio virtual, puesto que a veces se ven perdidos y para no fallar tratan de hacer todo sin detenerse a analizar por qué se hace.

Se concluye que la autorregulación en las competencias lectoras influye de manera dinámica y activa, llevando al estudiante a comprender por qué y para qué su proceso lector, y cómo construir aprendizajes significativos de manera autónoma, reconociendo el valor de la educación.

\section{Referencias}

Anaya, A. \& Anaya, C. (2010). ¿Motivar para aprobar o para aprender? Estrategias de motivación del aprendizaje para los estudiantes. Tecnología, Ciencia, Educación, 25(1), 5-14. https://www.redalyc.org/articulo.oa?id=48215094002

Arias, F. (2012). El proyecto de investigación: introducción a la metodología científica (6. ${ }^{\mathrm{a}}$ ed.) Episteme.

Bustamante. (2015, 21 de feb.). Niños colombianos pasan raspando en habilidad lectora: Las últimas pruebas Saber $3 .^{\circ}, 5^{\circ}$ y $9 .^{\circ}$ indican que hay pocos progresos en comprensión de textos. El Tiempo. https://www.eltiempo.com/ archivo/documento/CMS-15283357

Crispín, M.L., Doria, M.C., Rivera, A.B., Garza, T., Carrillo, S., Guerrero, L. et al. (2011). Aprendizaje autónomo: orientaciones para la docencia. Universidad Iberoamericana de México.

Goodman, K. (1986). El proceso de lectura: consideraciones a través de las lenguas y del desarrollo. En E. Ferreiro \& M. Gómez (eds.), Nuevas perspectivas sobre los procesos de lectura y escritura (pp. 13-28). Siglo Veintiuno. 
Hamui-Sutton, A. \& Varela-Ruiz, M. (2013). La técnica de grupos focales. Investigación en Educación Médica, 2(5), 55-60. http://www.scielo.org.mx/ scielo.php?script =sci_arttext\&pid=S2007-50572013000100009\&lng =es\&t $\operatorname{lng}=\mathrm{es}$.

Hernández, R., Fernández, C. \& Baptista, P. (2014). Metodología de la investigación (6a ed.). McGraw-Hill Interamericana.

Jiménez, E. (2014). Comprensión lectora vs competencia lectora: qué son y qué relación existe entre ellas. Universidad de Granada.

OECD. (2019), PISA 2018 Assessment and Analytical Framework. PISA, OECD Publishing. https://doi.org/10.1787/b25efab8-en.

Panadero, E. \& Tapia, J. A. (2014). ¿Cómo autorregulan nuestros alumnos? Revisión del modelo cíclico de Zimmerman sobre autorregulación del aprendizaje. Anales de Psicología, 30(2), 450-462. https://www.redalyc.org/ pdf/167/16731188008.pdf

Santrock, J. W. (2002). Psicología de la educación (1.a ed.). McGraw-Hill Interamericana.

Solé, I. (1992). Estrategias de lectura. Graó/ICE. https://www.uv.mx/rmipe/ files/2016/08/Estrategias-de-lectura.pdf

Torrano, F. \& González, M. (2004). El aprendizaje autorregulado: presente y futuro de la investigación. Electronic Journal of Research in Educational Psychology, 2(1),1-33. https://www.redalyc.org/articulo.0a? $\mathrm{id}=2931 / 293152878002$

Vargas, M. (2001). El docente: promotor de la lectura. Educere, 5(13), 24. https:// www.redalyc.org/articulo.0a? $\mathrm{id}=356 / 35601305$ 